\title{
Fetal Doppler for prediction of adverse perinatal outcome in preeclampsia in a low resource setting
}

\author{
Smriti Agrawal*, Vinita Das, Anjoo Agarwal, Amita Pandey
}

Department of Obstetrics and Gynecology, King George’s Medical University, Lucknow, Uttar Pradesh, India

Received: 25 July 2016

Accepted: 26 August 2016

\section{*Correspondence:}

Dr. Smriti Agrawal,

E-mail: smritijainagrawal@rediffmail.com

Copyright: (c) the author(s), publisher and licensee Medip Academy. This is an open-access article distributed under the terms of the Creative Commons Attribution Non-Commercial License, which permits unrestricted non-commercial use, distribution, and reproduction in any medium, provided the original work is properly cited.

\section{ABSTRACT}

Background: To assess the usefulness of fetal Doppler in predicting adverse perinatal outcome in preeclampsia.

Methods: All the women with preeclampsia from 30 weeks onwards were enrolled in the study. The umblical artery (UA) Systolic-diastolic (S/D) ratio>2standard deviation (SD) or UA-Pulsatility Index (PI) and UA-Resistive Index (RI) $>$ 2SD were taken as abnormal. The middle cerebral artery (MCA) was visualized and cerebroumblical PI ratio calculated. MCA-RI $<2$ SD was taken as abnormal. Adverse perinatal outcome was taken as major if there was stillbirth, immediate neonatal death, need of respiratory support and minor if neonatal nursery admission was done. Presence of acute fetal distress in labour was also noted.

Results: There were 107 women in the study. Major adverse outcomes were 11 . Umbilical artery-S/D ratio $>2$ SD (RR 4.46, 95\% CI 1.40-14.17) and RI >2SD (RR 3.36, 95\% CI 1.03-10.61) and MCA RI<2SD (RR 4.46, 95\% CI1.414.17) had a high relative risk to predict adverse major perinatal outcome. 39 babies were admitted in neonatal nursery and no parameters could predict them. Though UA-PI>2SD predicted acute fetal distress in labour (RR 2.33, 95\% CI 1.21-4.47), there was no association on multivariate regression analysis.

Conclusions: This study showed UA-S/D ratio and UA-RI $>2$ SD are significant predictors of perinatal deaths and immediate neonatal resuscitation in preeclampsia. Acute fetal distress in labour or neonatal nursery admission could not be predicted.

Keywords: Fetal distress, Stillbirths, Hypertension in pregnancy

\section{INTRODUCTION}

In preeclampsia there is increased uteroplacental resistance and reduced fetal perfusion due to inadequate invasion of spiral arterioles by trophoblast cells. This causes impaired fetal growth and fetal hypoxia. Precelampsia contributes to $25 \%$ perinatal morbidity and mortality and optimal evaluation of fetus is necessary to ensure a good outcome. ${ }^{1}$ Ultrasonography is done to evaluate fetal well-being by biophysical profile and recently Doppler ultrasound has emerged as a vital tool for antenatal surveillance. As the fetoplacental unit develops, impedance in umbilical artery decreases with increased diastolic velocity. The normal umbilical artery waveform in last trimester shows low impedance and high diastolic flow with low pulsatility index (PI) and low resistive index (RI). In pregnancies complicated with pre-eclampsia and IUGR, umbilical blood flow reduces due to placental vascular resistance. Doppler velocimetry shows elevated impedance to blood flow in placenta reflected by reduced or absent umbilical artery velocimetry and increase pulsatile index. ${ }^{2}$ In a study by Mendez L et al, it was seen that abnormal RI of umbilical artery and altered PI of umbilical artery were associated with preeclampsia. ${ }^{3}$

Presence of chronic fetal hypoxia leads to redistribution of fetal circulation to heart, kidneys and brain. Hence, compensatory vasodilation of middle cerebral artery with increase in diastolic flow results in a decrease in its 
pulsatility index (PI) and resistive index (RI) termed as "brain sparing effect". It is associated with low cerebral/umbilical PI ratio. Gramellini et al studied 45 normal growth and 45 growth retarded fetus and concluded that cerebral umbilical $(\mathrm{C} / \mathrm{U})$ Doppler ratio is usually constant during the last 10 weeks of gestation. In this study $\mathrm{C} / \mathrm{U}$ PI ratio $<1.08$ was taken as abnormal and predicted adverse perinatal outcome with $90 \%$ accuracy as compared to MCA PI or UA PI alone. ${ }^{4}$ Bano $\mathrm{S}$ et al also in a recent study stressed that $\mathrm{C} / \mathrm{U}$ PI ratio is a better predictor for adverse perinatal outcome. Apart from MCA-PI, MCA-PSV (peak systolic velocity) also holds promise. ${ }^{5}$ Middle cerebral artery is studied in growth restricted fetus and seen that high MCA-PSV predicts perinatal mortality better than a low MCA-PI in fetus with growth retardation that have abnormal umbilical artery Doppler. ${ }^{6,7}$

Umbilical vein pulsations and reversed flow in ductus venosus are reported as ominous signs of perinatal mortality and ventricular failure. ${ }^{8}$ In the analysis of Doppler velocimetry, some studies have shown that best prediction of perinatal outcome was achieved when umbilical artery absent or reverse diastolic flow was associated with reversal in ductus venosus and pulsatile umbilical vein blood flow. ${ }^{9}$

With so many indices in practice, there is still a dilemma about the best Doppler parameters. This study was planned to evaluate Doppler velocimetry in fetal vessels like umbilical artery, middle cerebral artery in women with preeclampsia and correlate with adverse perinatal outcome in a low resource setting.

\section{METHODS}

This study was conducted in the department of obstetrics and gynecology, King George's Medical University, Lucknow, U.P., India. The hospital is a tertiary institute with a 250 bedded department of obstetrics and gynecology. The protocol was approved by institution's ethics committee. It was a prospective observational study. All the women with preeclampsia from 30 weeks gestation onwards and willing to participate in the study were enrolled. A written informed consent was taken prior to enrolment. Women with known fetal congenital anomalies or diabetes mellitus were excluded from the study. The study was conducted over a period of 2 years from July 2012-2014. Gestational age was determined by best estimate from last menstrual period, clinical examination and if ultrasound for fetal biometry in early pregnancy was available. Preeclampsia was taken as blood pressure $\geq 140 / 90 \mathrm{mmHg}$ or more on two occasion at least 6 hours apart with proteinuria as per international society for the study of hypertension in pregnancy. ${ }^{10}$ Presence of intrauterine growth restriction (IUGR) was taken as estimated fetal weight on USG less than $10^{\text {th }}$ percentile for gestational age. Doppler velocimetry was done using Doppler ultrasound Toshiba model (Xario) with $3.5 \mathrm{MHz}$ curvilinear probe. An average of 3 consecutive Doppler velocity waveforms was used for statistical analysis. The angle between the ultrasound beam and direction of blood flow was maintained below 450 for umbilical artery. The umbilical artery was studied in a free loop. Umblical artery (UA) systolic diastolic ratio $>2$ SD above mean or absent or reversed end diastolic flow in umbilical artery was taken as abnormal. UA PI and UA RI were measured and value $>2$ SD was taken as abnormal. The middle cerebral artery was visualised at the circle of Willis and was insonated soon after its origin from internal carotid artery and the angle of insonation kept close to 00 . The pulsatility index was measured and cerebroumblical PI ratio calculated. MCARI was considered abnormal if measurement was $2 \mathrm{SD}$ below the mean. ${ }^{11}$ All the ultrasound evaluations were done by a single obstetrician with expertise in Doppler sonography. The intrapartum management of the women and the mode of the delivery were as per the hospital protocol. Acute fetal distress in labour (defined as abnormal fetal heart rate pattern and or presence of thick meconium in amniotic fluid) was noted. The team managing was aware of the antenatal surveillance result. The result of the last Doppler examination within 10 days of delivery was considered in the subsequent correlation with perinatal outcome.

\section{Adverse perinatal outcome was taken as major if}

- Intrauterine fetal demise.

- Immediate neonatal death.

- Neonatal intubation required at birth for resuscitation.

- Severe respiratory complication requiring assisted ventilation.

Adverse perinatal outcome was taken as minor if neonatal nursery admission for observation was done for complications like respiratory distress syndrome, metabolic complications etc.

\section{Statistical analysis}

Statistical analysis was performed using SPSS 16.0 software. Unpaired t-test was used to analyze continuous data. Categorical data was compared using Chi-square test. $\mathrm{P}<0.05$ was taken as statistically significant. Relative risk was calculated for abnormal UA PI, UA RI, UA S/D, MCA PI and cerebral-umbilical PI ratio. Multivariate regression was used to analyze effect of multiple variables.

\section{RESULTS}

There were 107 women enrolled in the study. Mean age of women was $27.1 \pm 4.05$ years. Of these $96(89.8 \%)$ women had preeclampsia, $11(10.2 \%)$ women had chronic hypertension with superimposed preeclampsia. Majority of women were either nullipara $(48.6 \%)$ or primipara $(33.6 \%)$. All the women were enrolled at 30 weeks onwards and umbilical artery and middle cerebral 
artery waveforms were recorded. Of 107 women, 79 (73.8\%) had mild disease and 28 (26.2\%) had severe disease at delivery. Abnormal umblical artery S/D ratio was seen in $31 / 110$ fetus $(28.2 \%)$. Umblical artery PI $>2$ SD was seen in $33 / 110$ fetus $(30 \%)$ and umblical artery RI> 2SD was seen in 38/110 fetus (34.5\%). Middle cerebral aretery RI < 2SD was seen in 46 fetus $(41.8 \%)$. The ratio of MCA and UA PI was abnormal in 42 fetus $(38 \%)$.

There were a total of 110 babies in the study including 3 sets of twins. There were $46(41.8 \%)$ babies with IUGR. Eleven major adverse outcomes were noted in the study2 stillbirths, 6 babies required immediate respiratory support and three babies had respiratory distress subsequently. Of the 6 babies who required immediate resusucitation, one expired in immediate neonatal period due to birth asphyxia (intrapartum fetal distress at 32 weeks in woman with severe preeclampsia), one expired after 10 days due to metabolic complications and the rest four survived. There were three babies who developed respiratory complication 24 hours after birth (not related to prematurity) and required respiratory support. All these 3 babies survived and were discharged subsequently. Of the two intrapartum stillbirths noted in the study, one was a severe IUGR at 30 weeks and the other had intrapartum fetal distress at 32 weeks leading to stillbirth. The abnormal waveform indices were compared with major adverse outcomes (Table 1).

Table 1: Relative risk of abnormal Doppler indices with adverse perinatal outcome.

\begin{tabular}{|lll|}
\hline Parameter & $\begin{array}{l}\text { RR }(95 \% \mathrm{CI}) \text { for } \\
\text { major adverse } \\
\text { outcome }\end{array}$ & $\begin{array}{l}\mathbf{R R}(95 \% \mathrm{CI}) \text { for } \\
\text { minor adverse } \\
\text { outcome }\end{array}$ \\
\hline $\begin{array}{l}\text { UA S/D ratio } \\
>2 S D\end{array}$ & $4.46(1.40-14.17)$ & $0.86(0.72-1.02)$ \\
\hline UA PI>2 SD & $2.80(0.91-8.53)$ & $0.91(0.79-1.00)$ \\
\hline UA RI>2SD & $3.36(1.03-10.61)$ & $0.89(0.78-1.03)$ \\
\hline $\begin{array}{l}\text { MCA } \\
\text { RI<2SD }\end{array}$ & $4.46(1.4-14.17)$ & $0.86(0.7-1.02)$ \\
\hline $\begin{array}{l}\text { MUPI } \\
<1.08(A B N)\end{array}$ & $0.35(0.11-1.13)$ & $1.04(0.92-1.18)$ \\
\hline
\end{tabular}

Since there were 2 stillbirths, and one baby died in immediate neonatal period, analysis for neonatal nursery admission is done in 107 babies. There were a total of 39 babies of 107 (36.4\%) who required nursery admission for observation either due to respiratory complications, metabolic complications or sepsis. The abnormal waveforms of umbilical artery and middle cerebral artery were also compared with neonatal nursery admission (Table 1).

In the study, $48(44.9 \%)$ women delivered vaginally and $59(55.1 \%)$ women had caesarean section. The most common indication for caesarean section was acute fetal distress $(32 / 59: 54.2 \%)$ followed by non-progress of labour (15/59:25.4\%). Of 46 babies with IUGR, 21 (45\%) developed fetal distress as compared 11 babies who had fetal distress without IUGR $(\mathrm{p}=0.04$; OR $=2.6,95 \%$ CI-1.06.9). When caesarean due to fetal distress (defined as abnormal fetal heart rate pattern and or presence of thick meconium in amniotic fluid) was compared with Doppler parameters, the relative risk with $95 \% \mathrm{CI}$ is shown in Table 2.

This result showed that only umbilical artery PI>2SD had a statistically significant correlation with acute fetal distress in labour (RR 2.33: 95\%CI 1.21-4.47). Rest of Doppler parameters did not have any significant correlation with acute fetal distress in labour. Since there was a statistical significant association between IUGR and acute fetal distress in labour, multivariate regression analysis was used to study the effect. It was seen that IUGR $(\mathrm{p}=0.2 ; \mathrm{OR}=1.98,95 \% \mathrm{CI}=0.67-5.8)$,Umbilical artery $\mathrm{PI}(\mathrm{p}=0.06 ; \mathrm{OR}=3.8,95 \% \mathrm{CI}=0.93-15.9)$, Umbilical artery $\mathrm{S} / \mathrm{D}(\mathrm{p}=0.8 ; \mathrm{OR}=0.86,95 \% \mathrm{CI}=0.19-3.89)$, and severity of disease $(\mathrm{p}=0.9 ; \mathrm{OR}=1.01,95 \% \mathrm{CI}=0.32-3.13)$ did not have any association with acute fetal distress in labour.

Table 2: Relative risk of abnormal Doppler indices with acute fetal distress in labour.

\begin{tabular}{|lll|}
\hline Parameter & RR $(95 \%$ CI $)$ & p \\
\hline UA S/D ratio $>$ 2SD & $1.35(0.67-2.69)$ & 0.06 \\
\hline UA PI $>2$ SD & $2.33(1.21-4.47)$ & 0.002 \\
\hline UA RI $>2 S D$ & $1.18(0.50-3.10)$ & 0.14 \\
\hline MCA RI $<2$ SD & $0.98(0.49-1.93)$ & 0.38 \\
\hline MUPI $<1.08(A B N)$ & $0.52(0.27-1.03)$ & 0.06 \\
\hline
\end{tabular}

\section{DISCUSSION}

Preeclampsia leads to increased perinatal morbidity and mortality due to associated IUGR and fetal hypoxia. Mendez L et al found that abnormal RI of umbilical artery and altered PI of umbilical artery and MCI were associated with preeclampsia, however this study did not analyse association of Doppler indices with adverse perinatal outcome. ${ }^{3}$ Fetal vascular changes on Doppler study can help us to identify the compromised fetus. There are various Doppler indices which have been studied to predict adverse perinatal outcome in postdated pregnacy, IUGR, preeclampsia etc but the results have been very contradictory. In this study fetal Doppler parameters in women with hypertensive disorder of pregnancy were studied and it was seen that umblical artery indices especially $\mathrm{S} / \mathrm{D}$ ratio $>2 \mathrm{SD}$ and $\mathrm{RI}>2 \mathrm{SD}$ along with brain sparing effect (MCA RI < 2SD) were associated with adverse perinatal outcome like stillbirth, neonatal death and need for immediate resuscitation. Neonatal nursery admissions were not associated with any abnormal Doppler indices. It is probably due to the fact that most of the nursery admissions were either due to sepsis or metabolic complications rather than hypoxia. 
Umbilical artery showed relevance in predicting adverse perinatal outcome. It was seen that abnormal umbilical artery blood flow depicted by elevted S/D ratio, high RI were associated with mojor adverse outcome. In a study by Ozeren et al all Doppler parameters were different in women with pre-ecampsia and umbilical artery S/D ratio showed a higher sensitivity and diagnostic accuracy $(88 \%$ and 94\%) in predicting adverse perinatal outcome as compared to cerebral-umblical ratio or other indices similar to our study. However in this study, intrapartum fetal distress and neonatal nursery admissions were all not analysed separately and thus difficult to comment upon. ${ }^{12}$ We suggest that the above two outcomes be analysed separately as the gravity of them is different from perinatal deaths and therefore cannot be clubbed. In another study by Yoon et al, umblical artery waveform was found to be a strong and independent predictor of adverse perinatal outcome in patients with preeclampsia $(\mathrm{OR}=14.2){ }^{13}$

Our study also found that cerebral-umbilical PI ratio $<1.08$ was not associated with adverse perinatal outcome. Cerebroumbilical PI ratio was proposed by Gramellini et al and he suggeted that MCA/umbilical PI ratio had higher diagnostic accuracy and sensitivity in detecting IUGR (70\%) and adverse perinatal outcome (90\%) as compared to individual PI of umbilical and middle cerebral artery. ${ }^{4}$ Similar to our findings, Ozeren et al also found that though cerebroumbilical PI ratio was higher in patients with preeclampsia, but had a lower sensitivity and diagnostic accuracy to predict adverse perinatal outcome. ${ }^{11}$ Lakhar et al also found that umbilical artery PI is more sensitive than cerebroumbilical PI ratio in predicting adverse perinatal outcome. ${ }^{14}$

\section{Doppler indices with acute fetal distress in labour}

This study showed that there was a significant association of IUGR and presence of fetal distress in labour. Most of the previous studies have not separately analyzed cesarean for intrapartum fetal distress with abnormal Doppler parameters and clubbed it with all adverse outcomes including perinatal deaths and nursery admissions. Martinez et al studied small-for-gestationalage group and concluded that middle cerebral artery vasodilation was associated with the highest risk of cesarean delivery $(\mathrm{P}<0.001)$ and cesarean delivery for nonreassuring fetal status $(\mathrm{P}<0.001)$ and also increased risk of neonatal acidosis (odds ratio, 9.0). ${ }^{15}$ In this study we found on univariate analysis that umbilical artery PI had a significant association with acute fetal distress in labour but on multivariate analysis, none of the variables including severity of disease, presence of IUGR or any Doppler indices had any significant association. Bano et al in a previous study found that umbilical and middle cerebral artery PI individually and cerebroumbilical PI ratio had significant association with cesarean section for fetal distress. However presence of confounders like IUGR was not analysed separately so it is difficult to comment if any other factors influenced fetal distress intrapartum. ${ }^{5}$ Since there are no clear Doppler parameters which suggest possible intrapartum fetal distress, it is suggested that all the women with preeclampsia in labour should be monitored vigilantly.

\section{CONCLUSION}

Doppler sonography is an indispensable tool in evaluating pregnancies complicated with uteroplacentral insufficiency. This study showed that of all the fetal Doppler parameters, umbilical artery-S/D ratio and umbilical artery-RI $>2$ SD are significant predictors of adverse perinatal outcome like perinatal deaths and immediate resuscitation. Umbilical artery-PI $>2$ SD was predictive of acute fetal distress in labour but on multivariate analysis failed to find any association. None of the Doppler parameters helped to predict neonatal nursery admission. Thus Doppler parameters can help the obstetrician and neonatologist to plan delivery and minimize adverse perinatal outcomes.

Funding: Intramural grant of King George's Medical University, Lucknow, UP, India

Conflict of interest: None declared

Ethical approval: The study was approved by the Institutional Ethics Committee

\section{REFERENCES}

1. Romero $\mathrm{R}$, Kalache KD, Kada N. Timing the delivery of the preterm severely growth restricted fetus: venous Doppler, cardiotocography on the biophysical profile? Ultrasound Obstet Gynacol. 2002;19:118-21.

2. Giles WB, Trudringer BJ, Baird PJ. Fetal Umbilical flow velocity wave form and placental resistance pathological co-relation. $\mathrm{Br} \mathrm{J}$ Obstet Gynacol. 1985;92:31-8.

3. Mendez MA, Gayta MV, Flores R. Doppler ultrasound evaluation in preeclampsia. BMC Res Notes. 2013;19:477.

4. Gramellini D, Folli MC, Raboni S, Vadora E, Merialdi A. Cerebral-umbilical Doppler ratio as a predictor of adverse perinatal outcome. Obstet Gynecol. 1992;79(3):416-20.

5. Bano S, Chaudhary V, Pande S, Mehta VC, Sharma AK. Colour Doppler evaluation of cerebral umbilical pubatility ratio and its usefulness in the diagnosis of intrauterine growth restriction and prediction of adverse perinatal outcome. Indian J Radiol Imaging. 2010;20(1):20-5.

6. Mari G, Hanif F, Kruger M, Cosmi E, Forgas SJ, Treadwell MC. Middle cerebral artery peak systolic velocity a new Doppler parameter in the assessment of growth restricted fetus. Ultrasound Obstet Gynacol. 2007;29(3):310-6.

7. Schenone MH, Mari G. The MCA Doppler and its role in the evaluation of fetal anemia and fetal growth restriction. Clin Perinatal. 2011;38(1):83102. 
8. Hecher K, Campbell S, Doyle P, Harrington K, Nicoladies K. Asessment of fetal compromise by Doppler ultrasound investigation of the fetal circulation. Circulation. 1995;91:129-38.

9. Baschat AA, Gembruch U, Weiner CP, Harman CR. Qualitative venous Doppler waveforms analysis improves prediction of critical perinatal outcome in premature growth restricted foetuses. Ultrasound Obstet Gynacol. 2003;22:240-5.

10. Brown MA, Lindheimer MD, Swiet M, Assche VA, Moutquin JM. The classification and diagnosis of the hypertensive disorders of pregnancy: statement from the international society for the study of hypertension in pregnancy (ISSHP). Hypertens Pregnancy. 2001;20:19-24.

11. Kurmanavicius J, Florio I, Wisser J, Hebisch G, Zimmermann R, Muller R et al. Refence resistance indices of the umbilical, fetal middle cerebral and uterine arteries at 24-42 weeks of gestation. Ultrasound Obstet Gynecol. 1997;10:112-20.

12. Ozeren M, Dinc H, Ekmen U, Senekayli C, Aydemir V. Umbilical and middle cerebral artery Doppler indices in patients with preeclampsia. Eur J Obstet Gynecol Reprod Biol. 1999;82:11-6.

13. Yoon BH, Lee CM, Kim SW. An abnormal umbilical artery waveform: A strong and independent predictor of adverse perinatal outcome in patients with preeclampsia. Am J Obs Gyn. 1994;171:713-21.

14. Lakhar BN, Rajagopal KV, Gourisankar PT. Ind J Radiol Imag. 2006;1:109-16.

15. Martinez R, Figueras F, Andrade HE, Oros D, Gratacos E. Fetal brain Doppler to predict cesarean delivery for nonreassuring fetal status in term smallfor-gestational-age fetuses. Obstet Gynecol. 2011;117(3):618-26.

Cite this article as: Agrawal S, Das V, Agarwal A, Pandey A. Fetal Doppler for prediction of adverse perinatal outcome in preeclampsia in a low resource setting. Int J Reprod Contracept Obstet Gynecol 2016:5:3439-43. 